\title{
Health Planning Requirements in Developing Countries
}

\author{
LLOYD FLORIO, M.D., Dr.P.H.
}

$D^{1}$ EVELOPING COUNTRIES is a disparate term that includes countries in such various stages of development as Taiwan and Laos. There is as much difference among them as there is between "developed" countries such as Australia or Japan. The developed countries have found it necessary to try to solve their medical manpower requirements in different ways, often with marked contrasts within a country itself. Australia, for example, has evolved different plans for rendering service to its densely populated southeast coast and its sparsely settled heartland. The same variability must be expected in the developing countries.

Some of the most important factors that must be considered are total population and population growth rate, density, size of area, urban and rural distribution of population, present physician-population ratios, presence

Dr. Florio is regional public health adviser of the Bureau for the Near East and South Asia, U.S. Agency for International Development, in Ankara, Turkey. He was chief public health adviser for AID in the Philippines for 7 years. This paper is based on a speech entitled "Medical Manpower Requirements in the Western Pacific Regional Office of the WHO," presented at the First Regional Conference on Education and Training: Conference of Deans of Medical Schools, Manila, November 1963. The opinions expressed are the author's and not necessarily those of WHO or AID. or absence of medical schools, ratios of other health personnel and hospital beds to population, birth and death rates, and causes of death. Although data are grossly inadequate in most developing countries, generally accurate impressions of disease problems are still possible. The educational attainments of the population are a matter of concern, for a nation must depend on high school and college graduates to become its technical personnel if we apply the same criteria that we do in Western cultures. The per capita income, purchasing power, gross national product, and collection of taxes determine the sums available for health services. Finally, one must be aware of the problems of language, cultural patterns, religion, geography, and infrastructure in constructing any plan for meeting manpower requirements, remembering that a country also has need for lawyers, engineers, and teachers, all of whom constitute a drain on the potential manpower pool.

The economic, cultural, sociological, technological, and ecological patterns of a society determine the applicability of health practices to that society. Transplants of whole technologies from one culture to another are not necessarily viable.

Progress in health is closely related to and largely dependent upon improvements in economic status and the educational level of the people. An educated populace with money will demand and pay for health care directly, or 
indirectly through taxes. In many instances as much health progress can be made through improved education and higher income as can be achieved by direct attack on health problems per se. This does not preclude the direct attack, for a person is often helpless in the face of certain health threats despite high educational or economic attainments. The individual person must depend, for example, on direct, concerted governmental action for ultimate and lasting protection against malaria. Such action is also the only means of breaking the circle of poverty, privation, and disability. It offers better opportunities for exploiting natural resources. The farmer might be able to supply his own potable water and approved means of sewage disposal; the urban dweller cannot.

Five questions should be posed and answered before it is possible to assess the medical manpower requirements of a developing country. The first four overlap and blend into each other.

1. How much can the country afford? How much of the available medical knowledge can the country use within the limitations of the funds available?

2. What will the people accept? Reject?

3. How much of the available budget should be devoted to prevention and how much to treatment?

4. Who will render the necessary services?

5. How are these determinations to be made?

\section{How Much Can the Country Afford?}

No country, developed or underdeveloped, is willing to spend enough to insure the application of available medical knowledge. There is always competition for the limited public and personal funds available. Education, public works, and defense, to name only three activities in the public sector, have a legitimate call on tax resources. Everyone argues that his particular activity is the most important, and someone or some group must ultimately establish priorities. How shall health expenditures be equated with national defense in the face of an imminent external threat? Is it not more important to promote economic development? Increased tax collections could then pay for services that cannot be afforded now. Families would also have more disposable income, some of which could be devoted to health purposes.
Needs as seen by experts are not necessarily equated with demands. It is a waste of money to supply a service for which there is little felt need. Nor is it always possible to supply a service with the sophistication that is possible in developed countries.

This leads to the closely related question of how much of the available medical knowledge a country can buy. A country may be able to afford much more than its officials think if they are willing to make some compromises. Several examples illustrate this point.

Corneal transplants have given sight to some people. Should an ophthalmic surgeon conduct a crusade to give sight to a handful of the blind? Could these funds not be better invested in trachoma control and vaccination against smallpox which will prevent much more blindness than the corneal transplants will cure?

Is it better to establish a simple routine for the diagnosis of tuberculosis, institute standardized home therapy, and carry out BCG immunization or build a tuberculosis hospital to do the complicated diagnostic studies and the surgical procedures required by the few? With severe limitations of funds and personnel, it may be better to close the tuberculosis hospitals already existing and use funds that maintain one bed to help instead 200 other people with tuberculosis. With this outlook, it is unnecessary to train as many chest surgeons and modern anesthesiologists with their expensive machines and anesthetic agents as would be needed to maintain sophisticated programs.

Are limited funds to be spent deworming a population? This is a relatively easy procedure, but a questionable and expensive one unless environmental changes that minimize reinfection also occur. Treatment for individual persons carrying intolerable worm burdens is, of course, always indicated.

\section{What Will the Population Accept?}

Even in highly developed societies with relatively unlimited medical resources, groups exist who do not accept modern medical practice and others that make extensive use of cultists and faddists. Some people use honey and applecider vinegar to replenish the mineral needs of the body. People in affluent societies abuse the use of vitamins and some depend on fake cancer 
and arthritis cures. Is it good to become dependent on tranquilizers, tonics, sedatives, and no-calorie colas? How progressive are the Westerners with their own cultism, faddism, and folkways?

Some culture-oriented therapies are at least as good as those that modern medicine has to offer. Medicine cannot yet offer a highly specific therapy for every specific disease. Mental illness is an example of this. If local therapy, even if it be incantations, results in a fair number of cures or amelioration of symptoms, and if scientific medicine produces no significantly higher percentage of cures, stay with the incantations. The people will anyway.

\section{How Much Effort to Devote to Prevention?}

Prevention, whenever this is possible, is the end to be sought. We must face the fact, however, that people are much more interested in receiving treatment for a disease they have now than preventing a disease they may or may not get in the future. The need for treatment is more visible and urgent and the outcome more dramatic. If the preventive and curative functions are vested in a single person, he will be forced to emphasize therapy. The astute will give the therapy and exploit the opportunity to promote prevention. In any event, health personnel must be prepared to consider a budget that favors treatment over prevention. This need not be regretted if therapeutic opportunities are exploited to emphasize prevention.

Medical schools, despite pleas to the contrary, emphasize diagnosis and treatment. Yet it is insufficient to say that medical schools in newly developing countries should emphasize prevention. Will the preventive measures be understood, can they be applied, can the country pay for them, and are they culturally acceptable and desired by the population, or must you first mold the society in the pattern of the society whose medical practice you wish to emulate? This is not always an impossibility, but it takes time, frequently generations of time. Age-old patterns obstruct the application of modern medical and preventive practices in most societies. You must either modify the practices to meet the situation, or remold the society, or do some of both. There is no answer, then, to the question of how many physicians and nurses are needed. The question that must be asked concerns the kind of medical manpower needed. The kind needed in one country may be nonexistent any place else in the world.

\section{Who Will Render the Necessary Services?}

It seems obvious that underdeveloped countries cannot exploit all the medical knowledge now available. It also seems obvious that many countries will have to be satisfied with a level of service given by persons with much less training than implied in the M.D., nursing, engineering, or paramedical degrees.

The World Health Organization cautions that even if a country can afford and could staff a medical school, internationally minimal standards for medical education must be met if the graduates of that particular school are to be admitted to more advanced studies abroad. Furthermore, physician graduates will not settle in the impoverished rural areas without adequate compensation and without medical or cultural facilities. Too many students in many countries enter medical school not to become members of a humanitarian profession, but to have greater social prestige, gain a higher income, and work in urban centers, if, in fact, they work in medicine at all.

Since the whole spectrum of medical and auxiliary medical personnel cannot be provided, much less trained, in all countries, we must be satisfied with a lower level of competence in many areas. As the country progresses and can afford and will utilize something better, ingenuity will be required to devise legitimate uses for personnel previously trained at lower levels of competence.

Nearly illiterate villagers with minimum training in the use of today's drugs can do more to save lives and cure or ameliorate disease in the developing countries than the best physicians could have done 25 years ago. The specifics, such as penicillin, available for most communicable or infectious diseases were not part of the physician's armamentarium 25 years ago. Illiterate villagers cannot do surgical operations, but the manpower and facilities needed for this type of curative medicine should enjoy a low priority compared with the treatment of diseases that are readily relieved with today's drug therapies. Such disease constitute most 
of the illnesses prevalent in underdeveloped rural areas.

Neither starry-eyed aims to ape the more developed countries nor misguided aspirations of the medical profession to maintain the standards of the profession should prevent the planning of the type of service that will achieve the greatest ultimate good for the people. Whenever the situation justifies it, we must train the best educated, most dedicated and willing local person who will be satisfied to work in that particular area to diagnose and use a standardized treatment for the diseases that represent the common causes of morbidity and mortality and forget the rest. Such a person would enjoy increased status and a higher income in his own community. He would be satisfied to remain there, whereas physicians and other highly trained medical personnel would probably have to be drafted for a period of mandatory service. I contend that highly trained medical manpower cannot be recruited voluntarily in adequate numbers for rural poverty-stricken areas. Lack of financial incentives, which neither the local nor national government can provide; inadequate medical facilities, such as hospitals; and cultural isolation make highly trained persons, physicians as well as others, shun the underdeveloped rural areas. This is seen even in the United States where conditions in rural areas are relatively ideal by comparison.

The staff to train these workers should speak the local language and be relatively well trained, but must especially have the insight to appraise the local cultural situation and provide a type of training suitable and acceptable to the people who are going to receive the service.

I share the sentiments expressed by Robert Morison, formerly with the Rockefeller Foundation, who has serious qualms about fellowship training abroad (1).

Heretical though it may be to say so, most fellowship training has been too exclusively focused on research with too little opportunity for learning such basic educational techniques as planning student laboratory work or conducting a proper clinical clerkship. Problems of administration are completely neglected.

By and large teachers in the United States know far too little about conditions in underdeveloped countries. They are therefore unable to provide visitors with the sort of experience that will be relevant to the task back home. Naturally enough, most of our capable teachers and investigators are concerned with extending the range of medical knowledge. Their emphasis is on the unsolved problems, many of which require expensive apparatus and much time and effort to attack. In the underdeveloped countries the major problem is one of using scarce resources as efficiently as possible to relieve the suffering, incapacity, and mortality caused by conditions which are already well understood.

\section{How Does a Country Determine Requirements?}

In order to determine a country's health planning requirements, the highest constitutional authority should appoint a top-level committee to determine the nature of the health problems of that particular society and the country's capacity for handling such problems, and to insure consideration and implementation of its recommendations. The committee should consist of the chief medical officer, a representative or two from suitable public or private international health agencies as consultants, several native sociologists or cultural anthropologists, the most hardheaded financial officer in the country, respected and influential legislative representatives of the major political parties, a demographer, and several medical and paramedical representatives whose experience and insight do not force them to follow patterns imposed by their particular professional affiliations. If the country has one or more medical schools, a highly influential representative from these schools should be included. The medical schools could not only help to motivate physicians toward rural practice, but could also train subprofessional people and even provide the necessary supervision and backstopping for them.

The difficult job will be to find such dedicated persons and convince them of the vitality of their mission, which is to define the problem, propose solutions, and dedicate themselves to the implementation of their proposals.

The committee must address itself to the following types of questions.

1. What priority can health expect in our particular situation? What is a realistic sum that can be made available for medical and preventive services?

2. What are the disease problems in the ap- 
proximate order of importance and priority? Which diseases are seriously hampering economic and social development? Which of these can the country tackle? How should the available funds be apportioned between curative and preventive services? Should we attempt to create new desirable demands? Can we finance them?

3. Is some type of population control program necessary, feasible, and culturally acceptable? Will such a program contribute significantly to the solution of our economic, social, and medical problems?

4. What types of personnel are going to be required to render these services? How will they be trained and at what cost from the funds available? What outside sources of help are available? How dependable are these sources over the long pull?

5. What plans can be made for providing a higher level of trained personnel in the future? To what use will personnel be put who have previously been trained at lower levels of competence?

6. What organizational pattern is required and feasible to implement present and future plans?
Each country must define its own problems and determine its own solutions. There should be no surprise, or regret, that proposed solutions differ from those of others. Unconventional answers are required, for it is abundantly evident that most of the conventional answers have not solved the problem.

\section{Summary and Conclusions}

Conventional solutions have not and will not solve the health problems of newly developing countries. Neither can these countries afford or even use the medical knowledge now available. A select group in each country must make a realistic determination of how much money is available for health, the purposes for which it must be spent, and the type of training that must be given to nonprofessional personnel to render the necessary services.

\section{REFERENCE}

(1) Morison, R. S.: International education exchange in medicine: Viewpoint of a private foundation. In Proceedings of the 58th Annual Congress on Medical Education and Licensure, Chicago, Feb. 4-5, 1962.

\section{Reorganization of the National Institute of Mental Health}

The National Institute of Mental Health, now a bureau of the Public Health Service, hopes to provide a more comprehensive and unified approach to the problem of mental illness as a result of its new status. It will remain the focal point for the nation's effort to improve the mental health of the people through development of knowledge, manpower, and services to provide and sustain mental health, prevent mental illness, and treat and rehabilitate mentally ill people.

The new bureau will continue to be known as the National Institute of Mental Health because it is widely known as the entity in the Federal Government concerned with mental illness and health. Headquarters of the bureau, which is organized into seven divisions and five offices, is located at 5454 Wisconsin Avenue, Chevy Chase, Md.

Mental health intramural research activities carried on in the Clinical Center are under joint administration of NIH and NIMH. A new division of special mental health research is located at St. Elizabeth's Hospital, Washington, D.C. The addiction hospitals of the Public Health Service at Lexington, Ky., and Ft. Worth, Tex., have been transferred to the NIMH and will become clinical research centers. 